\title{
Probing How Counterion Structure and Dynamics Determine Polyelectrolyte Solutions Using EPR Spectroscopy
}

\author{
Dariush Hinderberger • Hans W. Spiess • \\ Gunnar Jeschke
}

Received: 14 May 2009/Revised: 28 June 2009/Published online: 13 November 2009

(C) The Author(s) 2009. This article is published with open access at Springerlink.com

\begin{abstract}
In this review article, we describe how methods of electron paramagnetic resonance (EPR) spectroscopy were used to investigate polyion-counterion interactions in polyelectrolyte solutions. This subject is usually treated experimentally by light, X-ray, or neutron scattering techniques. It is shown that a large arsenal of EPR spectroscopic methods-from various sophisticated methods of line shape analysis of continuous-wave EPR, via electron spin echo envelope modulation, nanoscale distance measurements through double electron-electron resonance to high-field pulse EPR-can be used to characterize the intrinsically complicated structures formed in polyelectrolyte solutions. We show that even polymer physical models such as scaling relations can be tested in this way. The distinguishing feature with respect to the numerous scattering studies in this area is that EPR techniques are local methods, and by employing spin-carrying (i.e., EPR-active) probe ions, it is possible to examine polyelectrolytes from the counterions' point of view.
\end{abstract}

\section{Introduction}

Polyelectrolytes are macromolecular substances that are soluble in water or other ionizing solvents and dissociate into macromolecular ions that carry multiple charges (polyions) together with an equivalent amount of ions of small charge and opposite sign (Fig. 1). They play an important role in fields of scientific research as diverse as molecular biology and nanotechnology [1-6]. Many biological macromolecules such as deoxyribonucleic acid (DNA) or proteins are polyelectrolytes and

D. Hinderberger $(\bowtie) \cdot$ H. W. Spiess

Max Planck Institute for Polymer Research, Ackermannweg 10, 55128 Mainz, Germany

e-mail: hinderberger@mpip-mainz.mpg.de

G. Jeschke

Laboratory of Physical Chemistry, Department of Chemistry, ETH Zurich, 8093 Zurich, Switzerland 


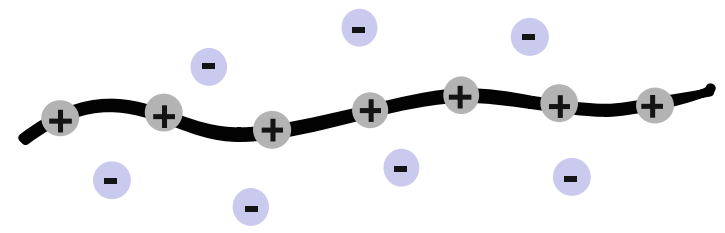

Fig. 1 Scheme of the "physicist's view" of a polyelectrolyte that is dissociated into a polyion and small counterions in polar solvents

use electrostatic interactions to trigger and control structural changes or binding of small molecules [5]. Synthetic polyelectrolytes are also applied commercially in cosmetics, fuel cells, and food and oil industry [7, 8].

Highly charged biological as well as synthetic polymeric materials have been under extensive experimental and theoretical investigations for several decades, and it is commonly acknowledged that their interesting structural properties stem from the delicate balance of two opposing interactions.

First, gain in entropy upon release of counterions leads to highly charged polymers, and electrostatic repulsion between the like charges on each repeat unit leads to a preference for extended conformations of the polyelectrolyte chain. Second, polyelectrolytes are usually dissolved in water or other solvents with high dielectric permittivity, which are poor solvents with respect to the polymer backbone (hydrocarbons). Poor solubility is equivalent to an attractive hydrophobic interaction between repeat units on the polyelectrolyte, which favors more collapsed structures [1].

Polyelectrolytes are probably the least understood class of macromolecules, which is remarkable when considering their importance in molecular biology and materials science. Despite numerous studies on polyelectrolytes, the combined effects of polyelectrolyte-counterion and polyelectrolyte-solvent interactions on polyelectrolyte structure are not fully comprehended. This is mainly due to the importance of a multitude of intertwined length scales in polyelectrolytes as compared with uncharged polymers, which makes theoretical and experimental investigations of the latter polymers much simpler [9]. It is known that the screening of intramolecular electrostatic repulsion by oppositely charged, in particular, multivalent, counterions can lead to a dominance of hydrophobic attraction and thus to more collapsed, globular chain conformations [10-12].

Characterization methods that probe macroscopic properties (such as conductivity) or can characterize long-range order (light-, X-ray-, and neutron-scattering) have been applied extensively in the past [13, 14]. Scattering experiments are usually carried out under addition of large amounts of inert salt (e.g., $1 \mathrm{M} \mathrm{NaCl}$ ), which effectively screens Coulomb interactions not only along one polyelectrolyte chain but also between different chains. This is necessary to damp intermolecular components of the scattering function. Information about chain conformations, such as the radius of gyration, $\left\langle R_{\mathrm{G}}^{2}>^{0.5}\right.$, or the mean-square end-to-end distance $<R^{2}>^{0.5}$ of chains can be gained by scattering methods, whereas a detailed, local picture of electrostatic interactions between polyion and counterions cannot be obtained. 
Magnetic resonance methods, such as electron paramagnetic resonance (EPR) spectroscopy [15] on spin-carrying counterions, being local, sufficiently sensitive and highly selective, have the potential to provide complementary information on microscopic structure and dynamics of polyelectrolyte materials [16, 17]. Ionic spin probes, which substitute for low-molecular-weight counterions, have been previously used to gain detailed information on structure and dynamics of polymer systems containing charges [16-26].

In recent years, together with EPR spectroscopy, other methods that were equally "exotic" in the realm of polymer characterization have been employed more extensively. This includes anomalous (resonant) small-angle X-ray scattering, small-angle neutron scattering, neutron-spin echo spectroscopy and pulsed-field gradient nuclear magnetic resonance, which can all probe, in particular, the interactions between counterions and polyions [27-29]. Altogether, with these new methods it was possible to examine a wide range of time and length scales and, to a large part, bridge the gap that still persists between theoretical description and experimental investigation of complex polyelectrolyte materials.

In this review, results from a variety of EPR methods, ranging from novel line shape analysis of continuous-wave (CW) EPR spectroscopy to modern pulse methods, such as electron spin echo envelope modulation (ESEEM) and double electron-electron resonance (DEER) are presented [30]. These methods elucidate interactions between several paramagnetic spin probes with multiple charges and oppositely charged polyelectrolytes in solution. These spin probes can be considered as tracers for multivalent counterions.

After a short introduction into two fundamental theoretical concepts for the description of polyion-counterion interactions, we will present experimental results from EPR spectroscopy and give (1) a qualitative, in large parts, model-free analysis of the spectroscopic findings, and (2) an analysis according to recent theoretical models of polyelectrolyte systems. It is shown that, in particular, the analysis with polyelectrolyte models can provide information on the radial distribution of counterions perpendicular to the chains, while changes in rotational dynamics of the counterions induced by interaction with the polyelectrolyte can be derived from the original data without treatment with such a model. We will also present how network formation by physical crosslinkers can be monitored and understood by high-field (W-band) pulse EPR spectroscopy. Finally, we will summarize our EPRbased insights into structure and dynamics of systems that are dominated by electrostatic interactions.

\section{The Physical Description of Polyion-Counterion Interactions}

\subsection{Counterion Condensation}

The concept of counterion condensation was introduced by Manning in 1969 [31, 32] and specified in 1979 [33], and is still the model most often used to describe the interaction of the ensemble of counterions with polyelectrolytes in solution. Within this model, the polyion is assumed to be an infinitely long rigid rod with equally 
spaced charges (spacing $b$ ). A fraction of counterions is electrostatically bound or condensed to the polyion, reducing the effective charge density on the rod-like chain, while the other fraction is unbound or free.

The distribution of counterions between the condensed and free states is determined by the interplay of opposing electrostatic (energetic) and entropic effects. The Coulomb energy decreases when the counterions are very close to the polyion chain, while entropy maximization counteracts the corresponding reduction of effective volume of the system and thus counterion condensation.

In a refined view of the condensed state, one may also differentiate between those counterions that form specific ion pairs with charges on the chain, so-called sitebound counterions, and such counterions that are trapped in the electrostatic potential around the polyion, so-called territorially bound counterions [33]. This is schematically depicted in Fig. 2.

For a quantification of counterion condensation, one needs to introduce an electrostatic screening length, the Bjerrum length $\lambda_{\mathrm{B}}[1]$ :

$$
\lambda_{\mathrm{B}}=\frac{e^{2}}{4 \pi \varepsilon_{0} \varepsilon k T} .
$$

The Bjerrum length is a specific function of the solvent permittivity and is the distance between two unscreened charges, at which the electrostatic interaction equals thermal energy $k T$. In water, at $298 \mathrm{~K}, \lambda_{\mathrm{B}}$ attains a value of $0.71 \mathrm{~nm}$.

The crucial parameter for counterion condensation is the ratio of Bjerrum length and charge spacing, called the Manning parameter [1]:

$$
\xi_{\mathrm{M}}=\frac{\lambda_{\mathrm{B}}}{b} \text {. }
$$

The linear charge density along the chain in the case without counterion condensation, $d_{0}=e / b$, is reduced to $d_{c}=\beta d_{0}$, if counterions are condensed to the chain, with $0 \leq \beta \leq 1$ being the fraction of free counterions.

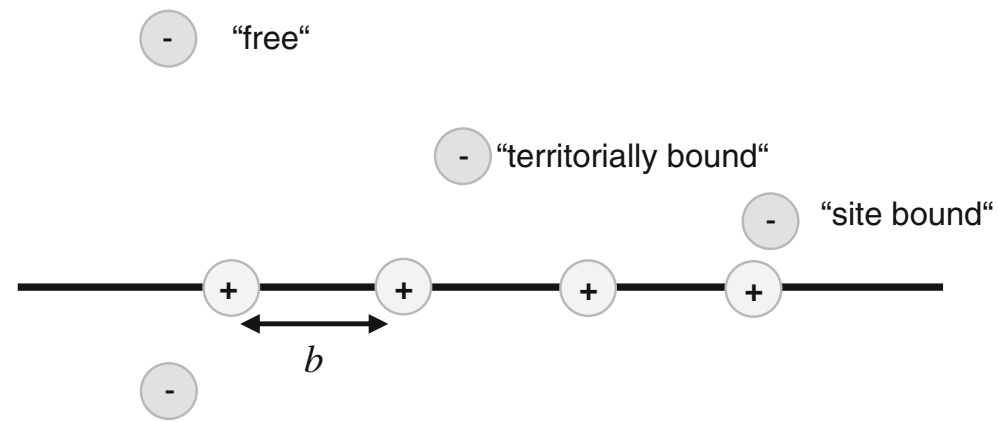

Fig. 2 Schematic illustration of the concept of counterion condensation. Excerpt of an infinite rigid rodlike polyelectrolyte chain and the three different counterion regimes following Manning. $b$ is the charge spacing along the chain 
Evaluation of the populations of condensed and free counterions leads to simple findings that correlate the Manning parameter to the onset of counterion condensation $[1,2]$ :

$$
\begin{aligned}
& \xi_{\mathrm{M}}<\frac{1}{z_{i}} \Rightarrow \beta \rightarrow 1, \quad d_{c}=d_{0} \\
& \xi_{\mathrm{M}} \geq \frac{1}{z_{i}} \Rightarrow \beta \rightarrow \frac{1}{\xi_{\mathrm{M}}}, \quad d_{c}=\beta d_{0}=\frac{e}{\lambda_{\mathrm{B}}}
\end{aligned}
$$

In Eq. (3), $z_{i}$ is the charge valence of the ions. Counterion condensation is expected for $\xi_{\mathrm{M}} \geq 1 / z_{i}$. From Eq. (3), another feature of counterion condensation becomes apparent: the condensation threshold decreases with the charge valence of the ions, so that multivalent counterions are always preferred to monovalent ions and may thus expel the latter from the condensed state. This is also plausible when considering the entropy of the overall system. If one $z$-valent ion is condensed, $z$ monovalent ions can be expelled, thus increasing entropy (number of individual particles) of the system. This effect has been utilized to probe polyelectrolytes by simply adding multivalent, EPR-active tracers for counterions.

Counterion condensation has been successfully used to interpret experimental data for DNA [34], but often fails to describe highly flexible polyelectrolytes. This is a consequence of the fact that the approximation of a polyelectrolyte chain as an infinite rigid rod is poor for very short or highly flexible chains. Since the interplay between chain conformation and electrostatic potential is highly nonlinear, it can only be introduced into a Debye-Hückel-like description by modifying artificially the linear charge density for each stage of condensation. Analytical and numerical solutions to the nonlinear Poisson-Boltzmann (PB) equation for infinite rod-like polyions suggest that they intrinsically possess the effect of counterion condensation $[1,35]$.

By adding large excess of inert salt (e.g., $\mathrm{NaCl}$ ), one can screen the electrostatic potential to such an extent that the polyion behaves like a neutral polymer and counterion condensation effectively ceases. A weak point of this treatment is that it is hardly possible to estimate the "cutoff distance" for the condensed state, i.e., the distance that marks the transition from a condensed to a free counterion state.

Despite all shortcomings of this simple approach, it is still the most widely used concept to estimate and characterize counterion-polyion interaction. Together with the distinction of site, and territorially bound states, on the one hand, and a "free" state, on the other hand, it will be consistently used in the explanation of experimental findings of this work.

\subsection{The Cylindrical Cell Model and Derived Models}

The cell model or more specifically the cylindrical cell model is one of the most thoroughly investigated models for the theoretical description of polyelectrolytes in solution.

This model is applied in many studies as it can be derived analytically from the nonlinear PB equation [1-3] and, alternatively, numerical simulations using sophisticated algorithms are possible. With this model, many of the observed and 
suggested effects of polyion-counterion interaction can be illustrated that go beyond the simple Manning picture.

As shown on the right-hand side of Fig. 3, the model for the polyelectrolyte system consists of a linear, rigid polyion that is surrounded by a cylindrical volume of the same length, which marks the region where counterions are considered condensed to the chains. The following assumptions are made [35]:

(i) high dilution of polyelectrolyte (i.e., only single chains),

(ii) the cylindrical volume (i.e., the radius must be selected) equals the overall volume that the polyelectrolyte has in solution, and

(iii) counter- and co-ions are distributed such that each cylinder is electroneutral.

The last point is true for most of the studies, but recently a modified version of the model has been developed by Deshkovski, Obukhov, and Rubinstein [36] that does not require electroneutrality and thus allows a fraction of counterions that do not interact with the polyion. This version is called the charged cylindrical cell model (CCCM). Although, as in the case of Manning condensation, this is a rather simple view of the polyelectrolyte system, there is a variety of typical polyelectrolyte characteristics that can be explained in such models.

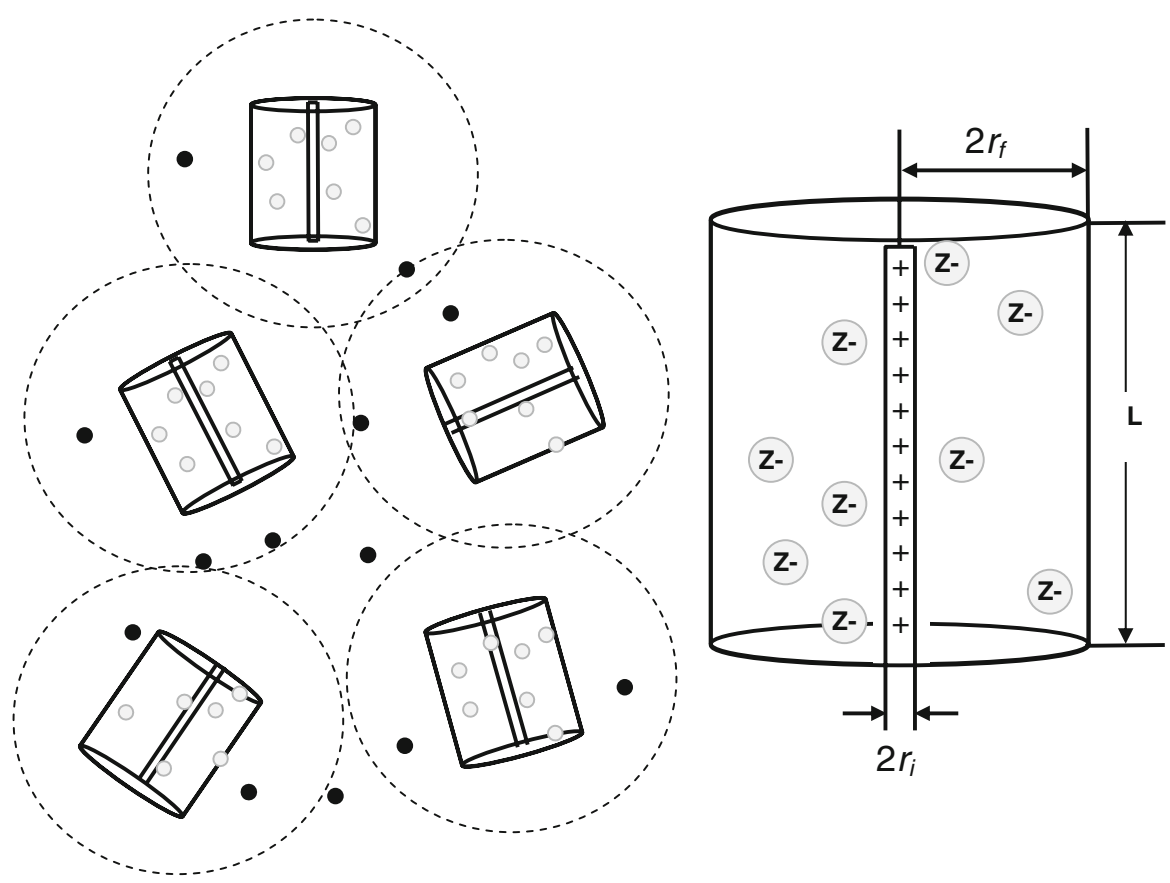

Fig. 3 Sketch of the cylindrical cell volume description for dilute solutions of rigid polyelectrolytes. $L$ is the chain length, $r_{\mathrm{i}}$ marks the initial and $r_{\mathrm{f}}$ the final radial coordinate of the cylindrical volume. Those counterions of charge $z$ that are within the cylindrical volume are shown in gray, those that are outside of this volume are drawn in black. The dashed circles around each cylinder indicate the spherical volume, in which the counterions are effectively unbound (no electrostatic interaction with the polyions). Note that cylinders need not be overall electroneutral. Adapted from Ref. [25] with permission from EDP Sciences 
One may assume counterions outside of the cylindrical volume as unbound and those inside the volume either site-bound or territorially bound.

To assess the quality of agreement of the predictions with experimental results, one has to always keep in mind that this model also starts off from PB theory, thus assuming weak electrostatic potentials, and that it effectively assumes a pure superposition of polymer and electrolyte behavior, which is inadequate. Despite these deficiencies, this is to date the most promising approach to describe polyelectrolyte-counterion interaction. A very detailed and thorough inspection of the cylindrical cell model can be found in Ref. [35].

The solution obtained by Deshkovski et al. [36] is strictly valid only for monovalent counterions, and they find that the natural boundary conditions for the general PB equation in their model are determined by two parameters. The normalized bare linear charge density along the polyion, $\gamma_{0}$, on the one hand, and the normalized net linear charge density of the cylinders, $\gamma_{R}$, on the other hand, govern the counterion distribution in the system. Deshkovski et al. [36] identify three distinct regimes (they denote them as phases, but the word regimes is probably more adequate) of charged chain-counterion distributions that are defined by $\gamma_{0}$ and $\gamma_{R}$, which all have in common that at intermediate radial distances $r$ from the chains $\left(r_{i}<r<r_{f}\right)$, the counterion concentration $c(r)$ shows a power-law dependence on the radial distance:

$$
c(r) \propto r^{-2 \gamma} .
$$

The scaling exponent $\gamma$ and hence the shape of the counterion distribution is different in each of these regimes. The resulting three regimes of counterion binding are:

Regime I: $\gamma=\gamma_{0}, \gamma_{0}<1$, "weakly charged polyions"

In this regime, the chain is only weakly charged, and counterion condensation as described by Manning has not yet commenced. By estimating the number of counterions $N$ at a typical length scale $r$ :

$$
N=2 \pi \int_{r}^{2 r} c\left(r^{\prime}\right) r^{\prime} \mathrm{d} r^{\prime} \propto r^{2\left(1-\gamma_{0}\right)},
$$

which increases with $r$, one can illustrate that most of the counterions in the cylindrical volume are far away from the charged central chain of the cylinders.

Regime II: $\gamma=1$, "saturated condensation"

In the second regime, the chain is strongly charged, and Manning condensation is fully active. Counterion concentration does not depend on charge densities $\gamma_{0}$ or $\gamma_{R}$ and obeys a simple $c \propto r^{-2}$ dependence. Estimation of the number of counterions $N$ at a typical length scale $r$ :

$$
N=2 \pi \int_{r}^{2 r} c\left(r^{\prime}\right) r^{\prime} \mathrm{d} r^{\prime} \propto r^{0}
$$

now gives a value independent of $r$. This regime is called saturated condensation, as moderate changes in the charge densities $\gamma_{0}$ or $\gamma_{R}$ do not significantly alter the 
counterion distribution in the cylinders. In the $\left(\gamma_{R}-\gamma_{0}\right)$ phase diagram of the calculated system (see Ref. [36]), this is the regime most likely attained by the system for intermediate to high linear charge densities on the chain $\gamma_{0}$ and low to intermediate charge densities on the cylinders $\gamma_{R}$.

Regime III: $\gamma=\gamma_{R}, \gamma_{R}>1$, "unsaturated condensation"

This regime is rather similar to regime II, Manning condensation is active but the concentration profile is much steeper (i.e., counterion concentration drops off even quicker than in regime II), which is obvious from the $c \propto r^{-2 \gamma R}$ dependence $\left(\gamma_{R}>1\right)$. The number of counterions $N$ at a typical length scale $r$ is given by:

$$
N=2 \pi \int_{r}^{2 r} c\left(r^{\prime}\right) r^{\prime} \mathrm{d} r^{\prime} \propto r^{-2\left(\gamma_{R}-1\right)} .
$$

Most of the counterions are very close to the chain and the remainder of the cylindrical volume is only sparsely occupied by the counterions.

This model will provide important information on the $r$-dependence of the counterion concentration by analysis of the broadenings in the CW EPR spectra of spin-carrying counterions in solutions of polyelectrolytes.

\section{EPR Spectroscopy on Polyelectrolytes}

\subsection{Studied Systems}

Out of the vast field of polyelectrolytes, we selected two distinct types of polymers. Scheme 1 displays the structures of two rod-like, self-assembling coordination polymers with $\mathrm{Ru}^{2+}$ as the charge-bearing group along the chain (RuCP) [37, 38]. The complex formation of $\mathrm{Ru}^{2+}$ ions and two terpyridine-based spacer molecules leads to octahedrally coordinated $\mathrm{Ru}$ centers with kinetically stable coordination bonds (for details on synthesis see Refs. [37, 38]). Several polymers of this kind with different organic spacer molecules and metal ions (especially of the Fe-group in the periodic table of elements) have recently been developed for two reasons. First, they are excellent model systems to probe solution behavior of polyelectrolytes as they retain their rigid extended conformation regardless of factors like ionic strength. Second, because of their regularly spaced transition-metal ions and fully conjugated $\pi$-electron spacers, there is potential interest in their electronic and optical properties and for catalysis. We used coordination polymers with two different spacer lengths between the $\mathrm{Ru}^{2+}$ centers, as shown on the right-hand side of Scheme 1.

Unlike conventional polyelectrolytes, RuCP are not soluble in water but soluble only in solvents, such as dimethyl formamide (DMF), dimethyl sulfoxide (DMSO), and dimethylacetamide (DMAc). This constituted a problem because of the poor solubility of the used anionic spin probe in these solvents. This problem was overcome by the use of ternary solvent mixtures that included water. 


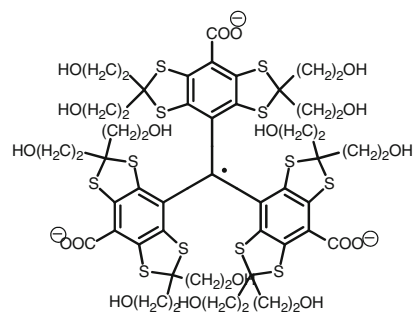

TAM

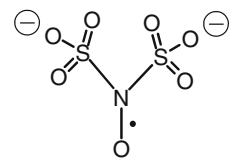

FS

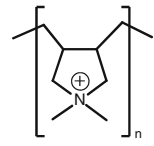

PDADMAC $\mathrm{n}^{*} \mathrm{Cl} \Theta$

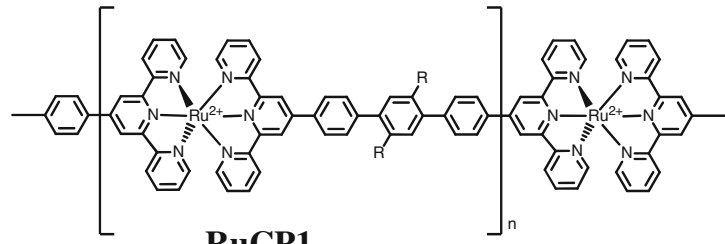

RuCP1

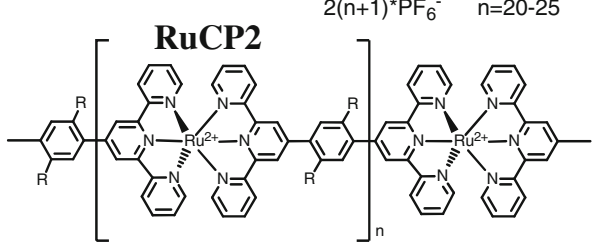

Scheme 1 Molecular structures of the charged spin probes and polyelectrolytes. Left Anionic spin probes: $\quad$ TAM $=$ triarylmethyl trianion, sodium salt; FS = Fremy's salt dianion (potassium nitrosodisulfonate). Right Cationic polymers; PDADMAC = poly(diallyldimethylammonium chloride); $\mathrm{RuCP}$, rod-like coordination polymers containing divalent ruthenium ions as charged groups. The number of repeat units $n$ is 20-25. Two different spacer lengths between the ruthenium centers are used. The side chains $\mathrm{R}\left(\mathrm{O}-\left(\mathrm{CH}_{2}\right)_{2}\right)_{2}-\mathrm{O}-\mathrm{CH}_{2} \mathrm{CH}_{3}$

In addition to the unusual rod-like RuCP, a commercially available flexible, unbranched polyelectrolyte PDADMAC [poly(diallyldimethylammonium chloride)] was investigated, which is also displayed in Scheme 1. The charged groups, namely, quaternary ammonium groups $\left(-\mathrm{NR}_{4}{ }^{+}\right)$, show a high degree of dissociation, making it a strong polyelectrolyte.

The cationic polyelectrolyte PDADMAC is extensively used not only in scientific research $[1,6,39,40]$ but also in industrial applications, e.g., to recover crude oil from oil/water emulsions. The used PDADMAC has a high molecular mass corresponding to a high degree of polymerization $\left(M_{\mathrm{W}} / M_{\text {monomer }} \sim 1500\right)$. The results for this polyelectrolyte can thus be compared to theoretical predictions for the long-chain limit.

The selection of charged spin probes used for EPR spectroscopy is presented on the left-hand side of Scheme 1. One can see that the spin probes belong to two different classes of paramagnetic species: (a) nitroxide radicals (Fremy's salt, FS) and (b) carbon-centered radicals (triarylmethyl trianion, TAM). The samples with variable concentrations of polyelectrolyte are prepared by varying the ratio $R$ of a spin probe (with the spin probe concentration always being held constant) to repeat units of polyelectrolyte: $R=c$ (spin probe) $/ c$ (polyelectrolyte), where, typically, the FS concentration is $0.5 \mathrm{mM}$ and the PDADMAC concentration is varied between 2 and $140 \mathrm{mM}$ in monomers, i.e., $0.0035<R \leq 0.25$. The dielectric properties and the viscosity of the solvents were varied using mixtures of water with glycerol (high 
viscosity), $N$-methylpropionamide (NMPA, higher dielectric permittivity than water) and ethanol (lower dielectric permittivity than water).

The charged spin probes simply constitute added salt since no original counterions of the polyelectrolytes are replaced by methods of ion exchange or dialysis. As explained before, for entropic reasons multivalent ions expel monovalent ions from the state "condensed" to the polyelectrolyte chains, so that the spin probe ions are expected to come into interaction distance with the oppositely charged polyelectrolytes by electrostatic self-assembly. In a simple picture, this can be anticipated by noting that multivalent ions reach the critical Manning parameter $\xi$ for counterion condensation already at lower values than monovalent ions.

\subsection{Localized Electrostatic Attachment of FS to Cationic Ru Centers}

The rigid rod polymers RuCP 1 and 2 are ideal model systems for charged polymers since they cannot change their conformation upon interaction with oppositely charged ions. We therefore first focus on these systems. Primary four-pulse DEER signals of glassy frozen solutions of FS with RuCP 1 and RuCP 2 are presented in Fig. 4a and c, respectively. The resulting distance distributions obtained from these data by a model-free direct integral transformation [41] both exhibit two marked peaks that are situated at 2.4 and $4.7 \mathrm{~nm}$ (RuCP 1, Fig. 4b) and 2.9 and $4.5 \mathrm{~nm}$

(a)

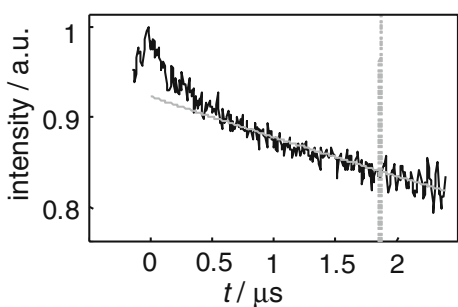

(c)

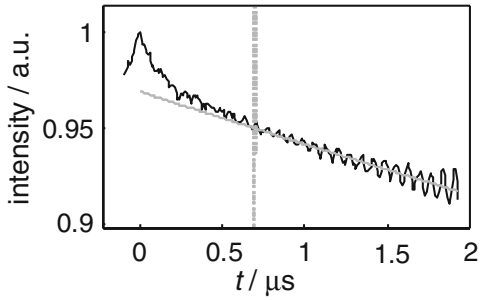

(b)

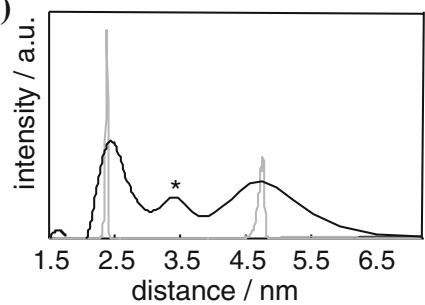

(d)

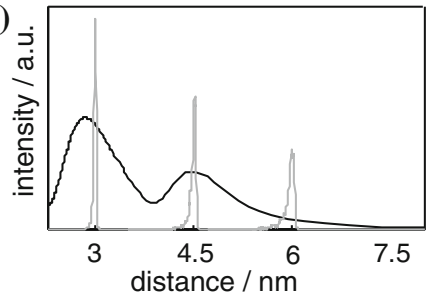

Fig. 4 DEER time-domain data (a, c) and extracted distance distributions (b, d) of FS in solutions of RuCP $1(\mathbf{a}, \mathbf{b})$ in $50 \mathrm{vol} \% \mathrm{DMF} / 50 \mathrm{vol} \%$ glycerol/water and RuCP $2(\mathbf{c}, \mathbf{d})$ in $33 \mathrm{vol} \%$ glycerol/67 vol\% water. The time traces were recorded at $T=20 \mathrm{~K}$. Gray lines are exponential fit to the data of FS/RuCP 1 between 1.9 and $2.4 \mu \mathrm{s}$ and FS/RuCP 2 between 0.7 and $1.8 \mu$ s (marked by the gray dotted line). The broad distance distributions (black lines) shown in b and $\mathbf{d}$ are obtained by integral transformation [41], the overlaid gray lines are $\mathrm{Ru}-\mathrm{Ru}$ distance distributions obtained from the molecular dynamics simulation as explained in the text. Amplitudes of the molecular dynamics distributions are scaled by a factor of 0.1. The asterisk in $\mathbf{b}$ marks an artifact due to simultaneous excitation of more than one pumped spin [59] or noise. Adapted from Ref. [24] with permission from Wiley-Vch 
(RuCP 2, Fig. 4d) and have approximately Gaussian shape. The additional weaker peak at approximately $3.5 \mathrm{~nm}$ in Fig. 4b (asterisk) may be a noise artifact, as it is not observed in data with shorter maximum dipolar evolution time (not shown). In the following, we nevertheless discuss the data for longer maximum evolution times, as they provide a better estimate of the width of the peak at $4.7 \mathrm{~nm}$.

Remarkably, in both cases, the two distances correspond rather nicely to the expected distances between $\mathrm{Ru}^{2+}$ centers, that are separated by one (peak at $2.4 \mathrm{~nm}$ for RuCP 1), two (peak at $4.7 \mathrm{~nm}$ for RuCP 1 and peak at $3 \mathrm{~nm}$ for RuCP 2) or three (peak at $4.5 \mathrm{~nm}$ for RuCP 2) spacers. The distance of $1.5 \mathrm{~nm}$ expected for $\mathrm{Ru}^{2+}$ centers separated by one spacer in RuCP 2 cannot be reliably detected, as it coincides with an artifact due to proton modulation [41, 42]. This proton modulation is mainly due to solvent protons. Differences in its depth may arise from the different solvent composition, but may also be caused by the slightly different timing of the two experiments. Distances longer than $5 \mathrm{~nm}$ cannot be extracted at the experimentally accessible maximum dipolar evolution times.

As it is known [43, 44], the modulation depth after dividing the data by the background function and renormalizing intensity at $t=0$, contains information on the fraction of FS ions that are close to the coordination polymer. We could estimate that less than $20 \%$ of the $\mathrm{Ru}^{2+}$ ions have FS counterions attached.

To obtain an estimate for the variation of the distances between $\mathrm{Ru}^{2+}$ centers, we performed molecular dynamics simulations of terpyridine end-capped oligomers of both compounds (RuCP 1: two repeat units and two endcaps, RuCP 2: four repeat units and two endcaps) in vacuum for a time of $2 \mathrm{~ns}$. The respective distance distributions are shown as gray solid lines in Fig. 4b, d. The theoretically predicted $\mathrm{Ru}-\mathrm{Ru}$ distance distributions are much narrower than the extracted FS-FS distributions, while the maxima in the distribution $(2.35 \mathrm{~nm}$ and $4.7 \mathrm{~nm}$ for RuCP 1; $3 \mathrm{~nm}$ and $4.5 \mathrm{~nm}$ for RuCP 2) are almost identical to those obtained from direct DEER transformation.

These DEER measurements are clear evidence for electrostatic attachment of at least a fraction of FS dianions to the charged groups on the coordination polymers. In the simple picture of (spherically symmetric) Coulomb interaction such pairing is favored compared to the interaction with the original monovalent counterions as it leads to a twofold increase in electrostatic interaction energy, and the replacement of monovalent by divalent counterions leads to a gain in entropy for the system as a whole [Eq. (3)]. Nevertheless, it is remarkable that electrostatic site attachment of counterions can be observed for relatively short, weakly charged polyions with approximately 25 repeat units and linear charge densities of the order of $1 \mathrm{~nm}^{-1}$.

The larger width of the peaks in the experimental FS-FS distance distribution compared to the peaks in the theoretically predicted $\mathrm{Ru}-\mathrm{Ru}$ distance distribution $(\mathrm{RuCP} 1$ : full width at half-maximum $(\mathrm{FWHM}) \approx 0.5 \mathrm{~nm}$ for the $2.4 \mathrm{~nm}$ peak and $\approx 1 \mathrm{~nm}$ for the $4.7 \mathrm{~nm}$ peak; RuCP $2: \mathrm{FWHM} \approx 0.6 \mathrm{~nm}$ for the $2.9 \mathrm{~nm}$ peak and $\approx 1 \mathrm{~nm}$ for the $4.5 \mathrm{~nm}$ peak) can be attributed to the sterical shielding of the $\mathrm{Ru}^{2+}$ ions by the terpyridine ligands and to the electrostatic nature of the localized counterion attachment. The upper part of Fig. 5 illustrates the geometry of counterion attachment for RuCP 1 as an example, assuming octahedral symmetry around $\mathrm{a} \mathrm{Ru}^{2+}$ center. At least four equal positions of closest contact for FS 


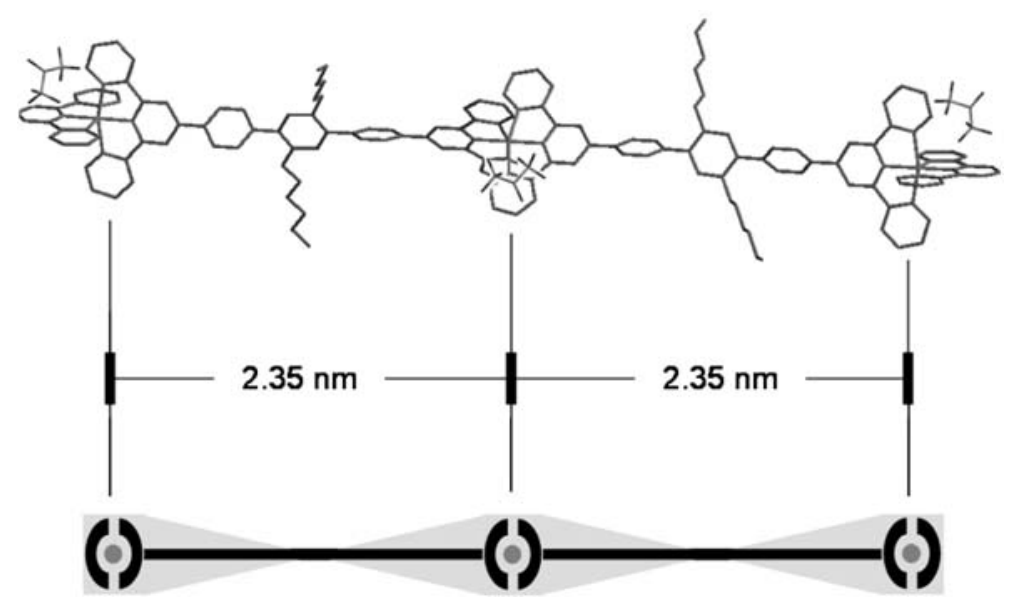

Fig. 5 Sketch of possible localized binding of FS ions to $\mathrm{Ru}^{2+}$ centers in $\mathrm{RuCP} 1$. Upper part Excerpt from the chain that has been geometry-optimized by force-field calculations (UFF 1.02) as explained in the text. The distinct distances between FS found from DEER data analysis $(\sim 2.4$ and $\sim 4.7 \mathrm{~nm})$ agree very well with the distances between $\mathrm{Ru}^{2+}$ centers separated by one or two organic spacers, respectively. Lower part Schematic plot of most probable attachment sites for FS ions (gray area). The relatively broad distribution of spin probe distances can be explained, if one accounts for the degrees of freedom of the electrostatic attachment. Adapted from Ref. [24] with permission from Wiley-Vch

counterions exist. The lower part of Fig. 5 is a schematic plot of the volume around RuCP 1, in which FS ions are most likely to reside. Force-field calculations of the structure of both coordination polymers suggest side lengths of $\sim 0.6 \mathrm{~nm}$ for the (hypothetical) square "box" that approximates the attachment volume around the $\mathrm{Ru}^{2+}$ site, i.e., the spatial region in which FS ions can access the oppositely charged $\mathrm{Ru}^{2+}$ ions. This attachment volume effectively translates to a degree of freedom for the electrostatic attachment of FS to $\mathrm{Ru}^{2+}$, which broadens the FS-FS distribution. Assuming a Gaussian distribution of ions in the attachment volume, such broadening has a square-root dependence on the number of spacers in between the $\mathrm{Ru}^{2+}$ centers, which is consistent with the observed increase in width of the FS distance peak when going to longer distances.

The electrostatic origin of the FS distance distribution also accounts for the fact that FS distance peaks are indeed Gaussian-like and not asymmetric as the theoretical Ru-Ru distance peaks (see Fig. 4b, d). The asymmetry of the latter peaks reflects that upon bending of an ideal rigid rod, the maximum distance must be that of full linear extension, which is at the same time the most likely distance. Broadening of the peaks by diffuse electrostatic attachment makes this effect unobservable in the FS-FS distance distribution.

\subsection{Dynamic Electrostatic Attachment}

In order to probe the dynamics of the observed attachment process in mixtures of FS and RuCP, CW EPR was performed on fluid solutions of the RuCP/FS samples. Like all nitroxide radicals FS exhibits a three-line CW EPR spectrum at ambient 
temperatures. It is well suited for the use as spin-carrying counterion as it is divalent, small and has very narrow lines, since there is no unresolved hyperfine coupling to intramolecular protons. In addition, the three FS lines are almost purely Lorentzian-like in shape except for a small deviation due to electrostatic repulsion between the like-charged FS ions $[45,46]$. The narrow Lorentzian lines have made FS a spin probe that has been used extensively in studies of rotational motion in CW EPR spectra [47].

In the RuCP/FS systems, the rotational diffusion of FS preferably takes place about an axis bisecting the angle between the two sulfonate groups (see Fig. 7b). Figure 6d shows the CW EPR spectrum of FS in the same solvent system without RuCP (black solid line). Comparison of the two spectra shows that the main difference lies in a change in the relative amplitudes of the three hyperfine lines upon addition of RuCP. In Fig. 6c and d, guiding lines connect the maxima and minima of the lines to make this change better recognizable. In spectra of pure FS, the center-field line is the narrowest and has the highest relative amplitude, followed by the low- and high-field manifolds. This pattern is also observed for pure FS in all other solvents. In the spectrum with $\mathrm{RuCP}$ added $\left(1: 1 \mathrm{Ru}^{2+}: \mathrm{FS}^{2-}\right)$, the low-field line is the narrowest and most intense, the center-field line is slightly less intense and the high-field line is significantly further decreased in intensity. This is clear evidence of the $\mathrm{Ru}^{2+}$ centers influencing rotation of FS and, in combination with the proof of direct electrostatic attachment of FS to $\mathrm{Ru}^{2+}$, one can safely assume that the local geometry of the attached state of FS looks as suggested in Fig. 7b. Unlike monovalent potassium ions, the divalent $\mathrm{Ru}$-ion seems to interact with both FS sulfonate groups simultaneously: this is energetically and entropically favorable. The lifetime of each individual "three-center ionic assembly", though, corresponds to fast exchange between the attached and detached state on the EPR time scale, as otherwise one would observe contributions of much slower FS ions in a bimodal (attached and detached) CW EPR spectrum, which is clearly not the case. In addition, the experimental EPR spectra with and without RuCP can be simulated very well with identical parameters except for the direction of the unique axis of the rotational diffusion tensor. Even the magnitudes of the rotational diffusion tensor elements are the same, which suggests that there is no significant impact on the local viscosity for the FS molecules and that only the local geometry of electrostatic interaction is changed.

When mixing FS as spin-carrying counterion with the flexible, highly charged PDADMAC polyelectrolytes, a new effect can be observed, namely, a marked increase in the peak-to-peak line widths. Again, like in the case of $\mathrm{RuCP}$, this is a clear sign of the dynamic attachment of a charged spin probe to the oppositely charged polymer, since EPR parameters such as line widths, line shapes, and hyperfine couplings are known to be very sensitive toward even small changes in the molecules' surroundings and rotational motion [48, 49].

Typical data for the probe FS in pure water and water/PDADMAC are shown in Fig. 6a. Unlike the spectra of FS/RuCP in Fig. 6c, d, with PDADMAC a marked increase in line width, in particular, at the flanks of the lines is observed. No impact of viscosity was found even for those samples containing the highest amounts of polyelectrolyte (spectra not shown). Hence, the impact of PDADMAC on the line 

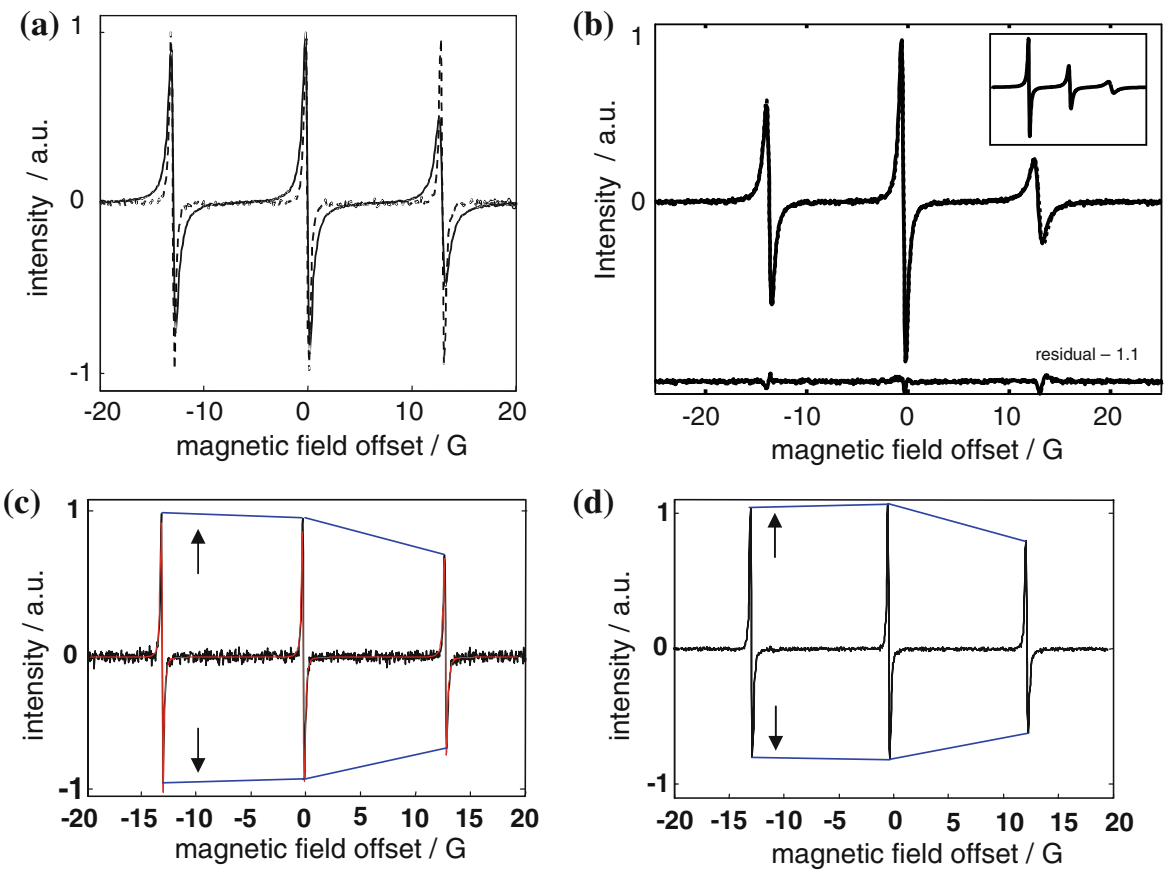

Fig. 6 X-band $(9.77 \mathrm{GHz}) \mathrm{CW}$ EPR spectra at $293 \mathrm{~K}$ of (a) FS in aqueous solution at two different spin probe/PDADMAC ratios $R$; (b) FS + PDADMAC (solid line, $R=0.011,46 \mathrm{mM}$ PDADMAC) in 70\% NMPA $/ 30 \%$ water. A simulation as described by Eq. (8) in the text, assuming preferred rotation about the axis indicated in Fig. 7a, is superimposed (dotted line) and the residual of the experimental and the simulated spectrum is shown. The inset shows simulation of the spectrum, assuming preferred rotation about the $C_{2}$ symmetry axis of the FS molecule; (c) $0.5 \mathrm{mM} \mathrm{FS}+0.5 \mathrm{mM} \mathrm{RuCP}$ (monomeric units) in $50 \mathrm{vol} \% \mathrm{DMF} / 50 \mathrm{vol} \%$ glycerol/water $(1: 1)$; (d) $0.5 \mathrm{mM} \mathrm{FS}$ in $50 \mathrm{vol} \% \mathrm{DMF} / 50 \mathrm{vol} \%$ glycerol/water (1:1); A spectral simulation of FS/RuCP according to the rotational diffusion tensor explained in the text and in Fig. 7 is shown in red. Lines connect the peaks of the measured spectra and are meant as guides to the eye. Arrows point to the difference in relative line intensities between the two cases. Adapted from Refs. [16, 22, 24] with permission from ACS Publishing and Wiley-Vch

amplitudes and line widths of FS EPR spectra is conclusive evidence that dynamic electrostatic attachment is the main cause of the observed spectral changes.

From the change of the EPR spectral line shape, one can not only show that FS is transiently attached to the polyelectrolyte, but also can again characterize the underlying attachment geometry. This is shown in Fig. 6b, where a typical CW EPR spectrum of FS + PDADMAC $(R=0.011)$ is presented together with a superimposed simulation (dotted line). To reproduce the rotational dynamics features on the basis the slow-motion theory of Schneider and Freed [22, explained further down in the text], a first rough preliminary fit (absorption spectrum) was then Fouriertransformed to give the time-domain data $V_{\text {sim }}(t)$, and a multiplication with an empirical broadening function (stretched exponential function) was performed to account for the observed concentration broadening:

$$
V(t)=V_{\text {sim }}(t) \cdot \exp \left(-(k \cdot t)^{x}\right),
$$


(a)

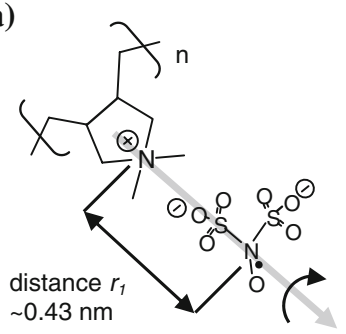

(b)

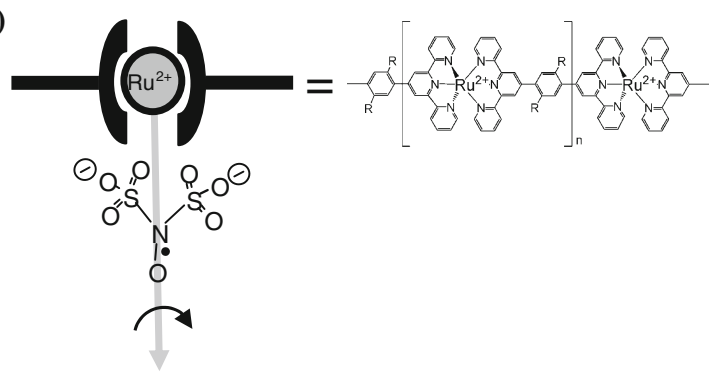

Fig. 7 Sketch of geometry and dynamics of FS counterions condensed to (a) a PDADMAC chain and (b) a rigid rod-like charged coordination polymer RuCP. a Sketch of the local geometry of an FS spin probe that is site-bound to a PDADMAC ammonium group. The gray arrow depicts the direction parallel to the axis of fast rotation of FS. The distance $r_{1} \approx 0.43 \mathrm{~nm}$ was obtained from ratio analysis of three-pulse ESEEM data as explained in the text [52]. Also shown is the rotational diffusion tensor underlying the simulation of experimental CW EPR spectra (Fig. 6b). The gray arrow marks the direction parallel to the axis of fast rotation; the axes of slow rotation are perpendicular to this axis. The rotational rates corresponding to the two principal rotations are $d_{\|}=3.7 \times 10^{9} \mathrm{~s}^{-1}$, and $d_{\perp}=5.5 \times 10^{7} \mathrm{~s}^{-1}$. b Visualization of the rotational diffusion tensor for FS with oppositely charged RuCP in the solvent system $50 \mathrm{vol} \% \mathrm{DMF} / 50 \mathrm{vol} \%$ glycerol/water (1:1). The gray arrow illustrates the principal axis of fast rotation of FS (15 tilted away from the NO-bond axis, in the plane spanned by S(1)NS(2)). The respective tensor elements of rotational diffusion are: $d_{\|}=30 \times 10^{9} \mathrm{~s}^{-1}$ (parallel to the unique axis in gray), $d_{\perp}=5 \times 10^{9} \mathrm{~s}^{-1}$ (perpendicular to the gray axis). In this case, the axis of fast rotation points along a bisecting line of the two charged sulfonate groups, which is suggestive of attachment to an ion along this direction. Adapted from Refs. [22, 24] with permission from ACS Publishing and Wiley-Vch

where $k$ is a characteristic decay time constant related to average concentration, and $x$ is the stretch factor characterizing the width of the distribution of concentrations. After inverse Fourier-transformation and pseudomodulation [50], a first-derivativemode spectrum was obtained. This convolution affects the narrower low-field and center-field lines more strongly than the high-field line that is already significantly broadened by slowed down rotational diffusion. The stretched exponential broadening function accounts for the distribution of local concentrations of the FS counterions, i.e., for the decrease of local concentration with increasing distance from the polyelectrolyte chain. This empirical broadening function is used as an approximation of a physically motivated broadening function that could be derived from the charged cylindrical cell model for the radial distribution function of counterions [22, 25, 36], and a calibration of the concentration dependence of the line width. The full analysis according to the physically motivated, radial distribution broadening function is presented in Sect. 3.5; here, we want to focus on the rotational dynamics. Note that the lines deviate strongly from a Lorentzian shape, as is manifested in the stretch factor $x=0.1$ of the empirical broadening function $\left(k=1.8 \times 10^{13} \mathrm{~s}^{-1}\right)$. Indeed, we found it impossible to fit the spectra assuming a single well-defined value for the Heisenberg exchange frequency in the simulation program [48]. Rotational diffusion can be described by an axial tensor with the unique axis along the N-S bond of FS as indicated in Fig. 7a. Rotation about this preferred axis is approximately 80 -fold faster than about axes perpendicular to it $\left(d_{\|}=3.7 \times 10^{9} \mathrm{~s}^{-1}, d_{\perp}=5.5 \times 10^{7} \mathrm{~s}^{-1}\right)$. These diffusion 
rates are approximately one $\left(d_{\|}\right)$and three $\left(d_{\perp}\right)$ orders of magnitude slower than for the almost isotropic rotational diffusion of FS in the same solvent mixture in the absence of polyelectrolyte [22]. The relative intensities of the three lines in the experimental spectrum clearly exclude an axially symmetric rotational diffusion tensor with the unique axis along the bisector of the two $\mathrm{N}-\mathrm{S}$ bonds, i.e., along the $C_{2}$ symmetry axis of the radical (see inset in Fig. 6b). Hence, the geometry of counterion attachment is clearly different from the one in the RuCP case.

Applying this combination of slow tumbling line shape simulation and convolution with an empirical broadening function, experimental spectra can be nicely fit throughout the whole range of polyelectrolyte concentration $(0.0036 \leq R \leq 0.125)$. While parameters $k$ and $x$ in Eq. (8) vary strongly (data not shown), which indicates substantial changes in local concentration of the radial distribution of concentrations, the rotational diffusion tensor is found to be almost independent of polyelectrolyte concentration. Over the whole range of concentrations studied here, the rotational diffusion rates decrease only by approximately $10 \%$ with increasing polyelectrolyte concentration. The stretch factor $x$ increases with decreasing polyelectrolyte concentration, indicating a more heterogeneous counterion distribution in dilute solution.

Since the CW EPR spectra of FS in fluid solutions of PDADMAC are governed by two features, rotational diffusion of FS spin probe and the heterogeneity of local concentration of FS, the convolution approach introduced here allows for a better discrimination of these two effects. The CW EPR spectra can be fully understood using the concept of counterion condensation (see Fig. 2 and Eqs. (1)-(3)). Figure 7 depicts the local geometry of the short-lived contact ion pair of FS and PDADMAC, as it is reflected in the rotational diffusion tensor. During the short time of the sitebinding, rotation around the electrostatic bond formed between ammonium and sulfonate group can still be fast, as the potential of the electrostatic bond is not significantly altered during the rotation and sterical hindrance may be weak. However, rotation perpendicular to this direction is slowed down strongly, as it is coupled to the collective motion of the polyelectrolyte chain. As derived from the spectral simulations described before, this slowing down of rotation about the axes perpendicular to the electrostatic bond during site-binding probably corresponds to a factor larger than 80 , as the PDADMAC chains are long (on average $\sim 1500$ monomeric units) and the polyelectrolyte rotational motion is slower than that of freely rotating FS by orders of magnitude. Because of rapid exchange of FS spin probes between the attached (site-bound) and detached (territorially bound) states, the rotational diffusion tensor is a time average over the almost isotropic reorientation of detached spin probes and the uniaxial reorientation of attached spin probes. The weak dependence of the rotational diffusion tensor on polyelectrolyte concentration indicates that the rate of this exchange between attached and detached states does not depend on $R$ for $R \ll 1$, i.e., for a large excess of polyelectrolyte repeat units with respect to the spin-carrying divalent counterions.

Such an analysis of the complex rotational dynamics imposed by dynamic electrostatic attachment in terms of Brownian diffusion with an axially symmetric rotational diffusion tensor amounts to a considerable simplification that comes at the expense of loosing some information on microscopic details of the process. Note, 
however, that within the regime of fast nitroxide dynamics observed here, the information content of the spectra is limited and a more complex dynamic model would lead to overfitting of the data.

An intriguing consequence of the good fit of the present data by the described dynamical model is that on the time scales governing the EPR line shapes, the sitebinding of the divalent FS spin probe mostly proceeds via only one of its sulfonate groups, not by forming a locally neutral aggregate with two repeat units. A model of fast hopping between two geometries of attachment involving both sulfonate groups can also be excluded, as it should again lead to an effective preferred rotation axis along the $C_{2}$ symmetry axis of the radical dianion, which is clearly at variance with the relative line intensities in the experimental spectra (Fig. 6b, inset).

Upon increasing the viscosity of the solutions significantly using high glycerol contents, we could slow down the attachment-detachment process and even characterize the combined motion of counterions attached to the polymer chains [21].

\subsection{Geometry of Counterion Attachment: Site-Bound and Territorially Bound Counterions}

To gain a deeper understanding of the site-attached state in PDADMAC-based polyelectrolytes we used three-pulse ESEEM, the stimulated echo-based version of ESEEM, a pulse EPR technique, on shock-frozen solutions of the studied systems.

The typical three-pulse ESEEM data for FS in the presence of PDADMAC show, after division of the time-domain signal by the stimulated echo decay (approximated by a stretched exponential function), maxima and minima of the modulation curve. In the Fourier transform of the time domain signal, sharp and narrow peaks were observed at a frequency of $1.1 \mathrm{MHz}$, which is the nuclear Zeeman frequency of a ${ }^{14} \mathrm{~N}$ nuclear spin at the static field $B_{0} \approx 3460 \mathrm{G}$ used in the experiments. In the absence of polyelectrolyte, no modulation was observed at this frequency. We therefore assign the measured modulation to the quaternary nitrogen in the PDADMAC repeat unit (Scheme 1; Fig. 7). The unusually narrow ${ }^{14} \mathrm{~N}$ ESEEM line is due to the nearly tetrahedral symmetry of the quaternary nitrogen, which leads to a small nuclear quadrupole coupling. Indeed, density functional theory (ADF 2002.01 package [51], BLYP density functional, TZ2P basis set) calculations of quadrupole couplings of quaternary ${ }^{14} \mathrm{~N}$ nuclei provide values of approximately $e^{2} q Q / h=200 \mathrm{kHz}$. Note that all data were recorded with $\tau=200 \mathrm{~ns}$, corresponding to a blind spot for the modulation due to the many very weakly coupled (hyperfine coupling $0.1 \mathrm{MHz}$ ), ${ }^{1} \mathrm{H}$ nuclei [30], which contribute to the so-called proton matrix line, so that modulation due to ${ }^{14} \mathrm{~N}$ nuclei dominates, which significantly simplifies data analysis.

When using ratio analysis, as it was pioneered by Ichikawa et al. [52], it is possible to extract quantitative information on the geometry of the complex obviously formed by FS and the PDADMAC repeat units.

The depth of the modulation measured in a three-pulse ESEEM experiment and its decay contain information on the electron-spin-to-nucleus pair correlation function. This information is conveniently extracted by ratio analysis, in which the 
ratio of the upper envelope (connecting the maxima of the modulation) to the lower envelope (connecting the minima of the modulation) is computed and then fitted to a simulated ratio. The simulation assumes that due to the $r^{-6}$ dependence of the modulation depth on the electron-nuclear distance $r$, the modulation is dominated by the shell of nuclei of the given isotope that is closest to the paramagnetic center. Modulation depth and decay depend on the radius $r_{1}$ of this shell, the isotropic hyperfine coupling $a$, and the average number $n$ of nuclei in the closest shell. With ratio analysis, we find an electron-nuclear distance of the closest approach of $r_{1} \approx 0.43 \mathrm{~nm}$, a virtually negligible isotropic hyperfine coupling of $a \approx 0.07 \mathrm{MHz}$, and an average number of $n \approx 0.20{ }^{14} \mathrm{~N}$ nuclei in the shell of the closest approach. Although this kind of data analysis is strictly valid only for spherical coordination shells and neglect of angular correlation [22, 30, 51], we may assume that it is a good approximation in the present case, where the number of nuclei at the closest approach for a given electron spin is usually zero or one, and one only has to deal with two interacting spins. Neglect of nuclear quadrupole coupling probably introduces an error mainly in $a$, but may also lead to a slight underestimate in $r_{1}$. For comparison, we performed force-field (MMFF94 force field in SPARTAN, Wavefunction, Inc.) calculations of two FS molecules and a short section of a PDADMAC chain with 12 monomeric units to obtain an estimate of an electrostatic closest approach. It is found that in such a model system in vacuum, the closest distance between the nitrogen atom of the FS nitroxide group and the chargebearing ammonium ion on the PDADMAC chain is approximately $0.4 \mathrm{~nm}$, and thus rather close to the values found from ratio analysis of ESEEM data.

With the results from ratio analysis of three-pulse ESEEM data, we have direct evidence for electrostatic attachment (site-binding) of FS to PDADMAC monomeric units. Together with the performed force-field calculations, the estimate of approximately $0.43 \mathrm{~nm}$ for the electron- ${ }^{14} \mathrm{~N}$ distance is a clear indication that contact ion pairs are formed, i.e., that for some counterions, no solvent molecules are located between the two oppositely charged moieties (ammonium and sulfonate groups). Figure $7 \mathrm{a}$ is a representation of the combination of this finding with the results for the rotational diffusion tensor of FS in PDADMAC. For all polyelectrolyte concentrations studied $(R \ll 1)$, the coordination number of ${ }^{14} \mathrm{~N}$ nuclei is approximately 0.2 . Hence, at a given time only $20 \%$ of FS spin probes form such contact ion pairs with PDADMAC chain monomer units, regardless of the ratio of spin probes to polyelectrolyte repeat units. Thus, Fig. 7a only gives a snapshot of the attached state in dynamic electrostatic attachment. Counterion attachment corresponds to the site-bound state in a dynamical equilibrium:

$$
\text { (free } \leftrightarrow \text { ) territorial binding } \leftrightarrow \text { site binding. }
$$

Dynamic information from CW EPR spectra in liquid solution indicates that the rate constant for the exchange between territorially bound and site-bound spincarrying counterions is significantly larger than $10^{9} \mathrm{~s}^{-1}$. If the lifetime of the sitebound (attached) state was of the order of a nanosecond or longer, we should observe bimodal spectra, i.e., a superposition of two dynamic components corresponding to territorially bound and site-bound FS probes. Furthermore, in the presence of polyelectrolyte, there is no spectral component with line widths and 
relative intensities, as they are observed in the absence of polyelectrolyte. This excludes the existence of a significant fraction of free divalent spin-carrying counterions. Such a picture, where at any given time all the FS counterions are either territorially bound or site-bound, is also consistent with the fact that the exchange between these two states is very fast, which requires that diffusion lengths are short. The picture is also in agreement with simple polyelectrolyte theory, which predicts our systems to be in the regime of saturated counterion condensation of divalent FS spin probes. From distance measurements with DEER [22], we could additionally assign a persistence length of individual PDADMAC chains of at least $5 \mathrm{~nm}$, the typical length scale for the DEER experiment. This means that on a length scale of at least $5 \mathrm{~nm}$, PDADMAC chains can be viewed as having an elongated, linear conformation, which becomes important for the modeling of the radial counterion distribution (RDC) in a cylindrical cell model (Fig. 3).

\subsection{Quantitative Description of the RDC: Line shape Analysis with CCCM}

As described before, in the CW EPR spectra in Fig. 6a and b, not only the rotational dynamics is changed upon addition of oppositely charged PDADMAC polymers, but also the lines are broadened, both, the peak-to-peak line widths as well as the flanks of the lines. These broadenings in fluid solution spectra can be assigned to exchange broadening of the spin probe ions and are a function of the frequency of molecular encounters of the spin probes. They thus contain valuable information on the local concentration of probe ions, and we have analyzed these broadenings not only using a stretched exponential broadening function (see Fig. 6b and Eq. (8)) but also using scaling predictions derived from the recently developed CCCM (Fig. 3).

For systems exhibiting counterion condensation, of the different regimes of counterion binding developed within the CCCM (Eqs. (4)-(7) and Fig. 3), the regimes of saturated condensation $(\gamma=1)$ and unsaturated condensation $\left(\gamma=\gamma_{\mathrm{R}}>1\right)$ are of interest. For saturated condensation, $c(r) \propto r^{-2}$ and the distribution is independent of the charge densities $\gamma_{0}$ and $\gamma_{R}$ throughout the whole cylindrical region. For unsaturated condensation, counterions are on average closer to the chain $\left(c(r) \propto r^{-2 \gamma}\right)$. These clear-cut predictions of scaling behavior result in quantitative predictions of the exchange-broadened EPR line shape and we have analyzed the set of CW EPR spectra of FS with variable concentrations of PDADMAC in three different solvents: pure water and mixtures of water with ethanol and NMPA, respectively.

The broadening function derived from the CCCM is introduced into line shape simulations in the time domain with the following equation:

$$
V(t) \propto \int_{r_{i}}^{r_{f}} r^{D-1} c(r) \exp \left[-\frac{c(r)}{c\left(r_{i}\right)} c_{0} t\right] \cdot \sum_{k=1}^{3} \exp \left[-\frac{t}{T_{2, k}}\right] \exp \left[i \omega_{k} t\right] \mathrm{d} r
$$

where $V(t)$ is the simulated EPR time-domain signal with $k=1$ corresponding to the low-field line and $k=3$ corresponding to the high-field line of the CW EPR spectrum of FS. $c_{0}$ is proportional to the maximum local concentration that is 
attained at $r=r_{\mathrm{i}}$ and $D$ is the dimensionality ( $D=2$ for cylindrical models). During the fitting, the $T_{2, k}, \omega_{k}$, and $c_{0}$ were allowed to vary. The $\omega_{k}$ account for changes in the microwave frequency and variation in the hyperfine coupling caused by the solvent, while the $T_{2, k}$ fit differences in the line widths of the Lorentzian contribution that are due to small variations of rotational dynamics with polyelectrolyte concentration [22]. Both the $\omega_{k}$ and $T_{2, k}$ vary only slightly during fitting and within a series of samples prepared with the same solvent. For a given function $c(r), c_{0}$ is the only parameter influencing the shape of the individual lines. The model parameter $r_{i}$ was fixed at $r_{i}=0.4 \mathrm{~nm}$ based on the distance for contact ion pairs of FS and PDADMAC, as found in the above-described ratio analysis of ESEEM data (Fig. 7a). The value for $r_{f}$ was fixed at $25 \mathrm{~nm}$; as for $r_{f}>15 \mathrm{~nm}$, the line shape does not depend on $r_{f}$. In Eq. (9), the time-domain signal for a single line with resonance offset $\omega_{k}$ and characteristic transverse relaxation time $T_{2}$ in the absence of exchange broadening, is now given by the second factor on the righthand side, a single-exponential decay function. Exchange broadening is introduced by integration over the pre-exponential factor $r^{D-1}$ multiplied by the exponential function that converts the $r^{-2}$-dependence of the local concentration into a Lorentzian line broadening in the limits from $r_{i}$ to $r_{f}$ (see Fig. 3). This is the first factor on the right-hand side of Eq. (9).

The CW EPR spectrum is then obtained by Fourier transformation of the sum signal $V(t)$, and subsequent pseudomodulation [50] with the same modulation amplitude as used in the experiments.

At moderate polyelectrolyte concentrations $(c($ PDADMAC $)=13.9 \mathrm{mM}$, $R=c(\mathrm{FS}) / c(\mathrm{PDADMAC})=0.036)$, the line shapes are very nicely fitted by the CCCM with $\gamma=1$ for all three employed solvents. The results are self-consistent with the integration limits, i.e., the radial distribution of counterions levels off at approximately $r=4 \mathrm{~nm}$, so that at the upper limit $r_{f}=25 \mathrm{~nm}$, a vast majority of counterions are accounted for. For the mixtures of water with ethanol and NMPA, fits of similar high quality are obtained throughout the concentration range $0.0035<R<0.13$, keeping $c(\mathrm{FS})=0.5 \mathrm{mM}$ fixed. In contrast, spectra at low concentrations of PDADMAC in pure water $(R>0.06)$ exhibit line shapes with strongly broadened outer flanks and a very narrow central part, which cannot be fitted by the CCCM with $\gamma=1$.

The sensitivity of the EPR line shape to the choice of $c(r)$ could be tested by fitting EPR spectra with different theoretical models for a low polyelectrolyte concentration in water/ethanol (central line of CW EPR spectrum shown in Fig. 8a). A poor fit is obtained for $c(r)=\exp [-\mathrm{k} r]$ (root-mean-square error, 0.789). Thus, a simple exponential radial counterion distribution is inconsistent with the data. In contrary, distributions of the type predicted by the CCCM are in accord with the EPR line shapes. Variation of $\gamma$ provides the best fit for $\gamma=1.021$ (root-meansquare error, 0.382) in water/ethanol and $\gamma=1.000$ in water/NMPA at $R=0.067$. For $\gamma<1$, the fits deteriorate rapidly, confer the residual for $\gamma=0.6$ (root-meansquare error, 0.462) in Fig. 8. This demonstrates that in the solvent mixtures, the line shape is very close to the one expected for saturated condensation, but as the fit quality deteriorates only slightly when increasing $\gamma$ beyond 1 , the data are consistent with both Manning-type condensation regimes in the CCCM, saturated $(\gamma=1)$ and 

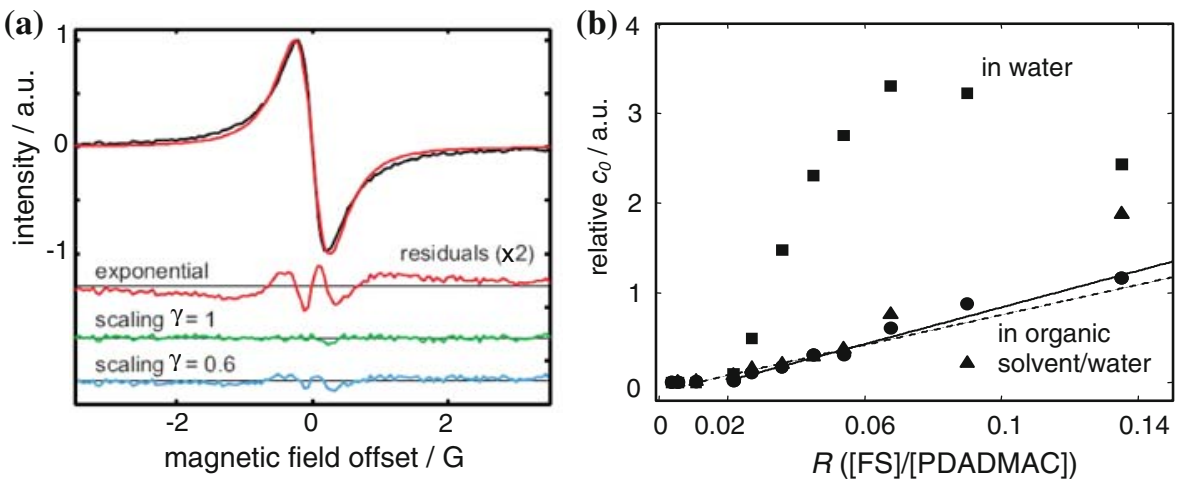

Fig. 8 a Fits of the central line of the CW EPR spectrum (at $\approx 9.7 \mathrm{GHz}, 293 \mathrm{~K}$ ) of FS + PDADMAC in water/ethanol at low polyelectrolyte concentration $(R=0.067)$ by different models for the RDC. Experimental spectrum and best fit by an exponentially decaying RDC. The three bottom traces a residuals multiplied by a factor of two of best fits by an exponentially decaying RDC, the CCCM with $\gamma=1$, and the CCCM with $\gamma=0.6$. b Dependence of parameter $c_{0}$ in Eq. (9) on the relative concentration $R$ of spin-carrying counterions with respect to polyelectrolyte repeat units, assuming saturated counterion condensation $(\gamma=1)$. Filled squares, in water; filled triangles, in NMPA/water; open circles, in ethanol/water. Linear regression fits for water/NMPA (solid line) and water/ethanol (dashed line) are also shown. Adapted from Ref. [25] with permission from EDP Sciences

unsaturated $(\gamma>1)$ condensation. Note that the dimensionality $D=2$ of the counterion distribution, inherent in the cylindrical symmetry of the model, cannot be determined ab initio. We find that variations in $D$ can be compensated, to some extent, by changes in $c_{0}$. Hence, the data do not allow for an independent determination of both the dimensionality and the scaling law.

Interestingly, for low concentrations of PDADMAC in pure water, fits remain poor, when $\gamma$ is allowed to vary. The data are thus inconsistent with unsaturated counterion condensation in the CCCM. The best fit for $c(r)=\exp [-k r]$ is even worse (root-mean-square error of 1.371 compared to 0.798 for $\gamma=1$ in the CCCM). One is thus forced to assume that at least one of the assumptions of the cylindrical models breaks down in this case. A possible explanation is a counterion-induced local coiling of the chain at the higher relative counterion concentrations. These findings indicate that in water, unlike in solvent mixtures, linear chain segments do not extend over a few nanometers in the presence of a moderate concentration of divalent counterions. This does not necessarily imply a collapse of the whole chain.

Consistency of the model with the data further requires that $c_{0}$ in Eq. (9) is directly proportional to the normalized concentration $R$ of spin-carrying counterions. For the mixtures of water with ethanol and with NMPA, the data, indeed, exhibit the expected linear dependence with zero intercept (Fig. 8b, experimental intercepts are -0.2 and -0.09 arbitrary units, respectively). In contrast, for pure water we find a strong deviation from this expected behavior for $R>0.022$. This is further proof for inconsistency of the data for water with predictions by the CCCM, especially for $R>0.06$. Parameters of the CCCM could also be varied by the addition of salt. In such experiments we found the expected decrease in the influence of the polyelectrolyte on the EPR line shape [16, 22]. With increasing salt 
concentration in water, deviations of the line shape from predictions by the CCCM become smaller, but at the same time, the Debye length approaches the length of the polyelectrolyte chain, thus invalidating one of the model assumptions. Therefore, we refrained from a quantitative analysis of these spectra.

EPR spectra of spin-carrying counterions in PDADMAC solutions agree with the scaling behavior of the RDC predicted by the CCCM for mixtures of water with ethanol or NMPA, corresponding to media with lower and higher mean permittivity than pure water, respectively. Any other model that arrives at a scaling prediction for the RDC close to $r^{-2}$ would be as good a description of our data. For pure water, however, the spectra clearly do not agree with such models. Whether this is due to an influence of water structure on the RDC or due to specific solvation in solvent mixtures cannot be deduced from our data. Independent of their origin, the clearly different experimental line shapes in water compared to the two mixtures demonstrate that mean permittivity is not sufficient to characterize a solvent mixture. As a limitation of our approach, we noticed systematic line shape deviations in high-viscosity solvents such as mixtures of water and glycerol, which probably result from incomplete averaging of magnetic dipole-dipole interactions between spin probes.

On a more general level the successful use of the predictions of the CCCM demonstrated that line shape analysis of CW EPR spectra of nitroxides in solution is a viable technique for tests of scaling approaches. If the dominating relaxation mechanism of the electron spins is known, as is the case here, such line shape analysis is analogous to analysis of dynamic light scattering data in terms of a distribution of decay times but relies on a different contrast mechanism and is sensitive to a shorter length scale. It thus complements dynamic light scattering studies in solution. EPR validation of scaling may be most useful for semi-dilute solutions of macromolecules in low-viscosity solvents, where established techniques often tend to fail.

\subsection{Network Formation Induced by Trivalent Star-Like TAM Counterions}

We extended our approach of characterizing polyelectrolyte interaction with paramagnetic counterions toward interaction of PDADMAC with large, organic, trianions of the triarylmethyl class (TAM, Scheme 1) [53, 54]. Unlike the divalent FS ion that is a rather small inorganic molecule, the TAM trianion represents a class of rigid, triangular-shaped organic molecules, with their structure deviating strongly from that of usual polyelectrolyte counterions such as chloride ions. In numerous studies such large organic molecules have been shown to interact with the oppositely charged polyion not only by electrostatic but also by hydrophobic interactions [55]. TAM, in particular, consists of three aromatic structural units and of 12 short hydroxyethyl moieties, which all might seek interaction with large organic macromolecules. The well-defined, rigid structure of this molecule and especially the triangular arrangement of charges with an intercharge distance of $1 \mathrm{~nm}$ make it a structural guiding block for supramolecular assemblies.

With a convolution approach mathematically similar to that described by Eq. (8) for FS fluid solution CW EPR spectra, we analyzed a series of samples, containing 
the TAM trianions and PDADMAC polyelectrolyte in the range of concentration ratios $R(R=c(\mathrm{TAM}) / c(\mathrm{PDADMAC}))$ between 0.0028 and 0.1 . The major difference to the above-described procedure was that we used low-temperature $(80 \mathrm{~K})$, electron spin echo (ESE)-detected EPR at high field/high frequency (W-band, $94 \mathrm{GHz}$ ). Using high-field EPR, one can resolve the very small anisotropy of the $g$-tensor [56] at least a little better than at standard X-band frequencies.

The measured spectra showed clear $R$-dependent line broadenings that increased with increasing $R$. These ESE-detected EPR spectra were fitted by a convolution approach, wherein optimized broadened spectra $V_{\text {sim }}\left(B_{0}\right)$ were simulated by convolution of a reference spectrum $V_{\text {ref }}\left(B_{0}\right)$ with a broadening function that accounts for the dipolar broadening. This approach is similar to the one discussed above for liquid solutions, where the broadening is due to exchange coupling. With this procedure, information on the distribution of distances between TAM trianions could be extracted: the dipolar broadening function corresponds to a Gaussian distribution of distances with two parameters: (a) the mean distance $\left(r_{\mathrm{dip}}\right)$ between unpaired electrons (centers of TAM ions), and (b) the width of the Gaussian distribution $\Delta r_{\text {dip. }}$. We found two types of TAM ions and simulated the experimental spectra by superposition of two spectral components, referring to the two fractions of counterions, clustered (dipolar broadened) and dilute (reference). The parameters $\left(r_{\text {dip }}\right), \Delta r_{\text {dip }}$, and the relative contribution of the respective fraction of TAM ions were fitted by minimizing the root-mean-square deviation between $V_{\text {sim }}\left(B_{0}\right)$ and the experimental spectra. This leads to a surprising result. We find that throughout the range $0.01<R<0.1$, the mean distance between an observer TAM trianion and its closest neighbor trianion decreases only slightly from $\sim 1.6$ to $\sim 1.4 \mathrm{~nm}$, and even for the highest polyelectrolyte concentration, the distance is still as short as $\sim 2.0 \mathrm{~nm}$. A change in polyelectrolyte concentration results mainly in a change of the fraction of trianions that are situated within clusters. Even at high dilution of TAM ions in PDADMAC (i.e., low $R$, high PDADMAC content), there is a nonnegligible fraction of TAM ions that are clustered.

The finding that mainly the fraction of clustered ions increases led us to propose a "zipper-like" crosslinking that resembles cooperative binding. Once one junction, i.e., several PDADMAC chain segments held together by TAM ions, has been established, other favorable sites for attachment are preformed. The effect arises from the proximity of the chains after formation of the first physical crosslink, but may be enhanced by a geometrical predisposition to form new connections: an isolated polyelectrolyte chain is expected to be locally stretched due to electrostatic repulsion and may thus act as a topological constraint. A possible mechanism of such cooperative binding at a given ratio $R$ is schematically depicted in Fig. 9.

This zipper-like network formation can be discussed in terms of entropy and enthalpy gains and losses. In general, electrostatic attachment increases entropy by the release of a larger number of monovalent counterions. In the case at hand, this entropy gain is partially offset by the entropy loss due to the loss of conformations of the polyelectrolyte chains. An unfavorable enthalpy increase is expected due to the approach of two chains or chain segments with total positive charges. This is counteracted by partial charge compensation by attachment of trianions and possibly by the preferential interaction of hydrophobic nature of the voluminous 
(a)

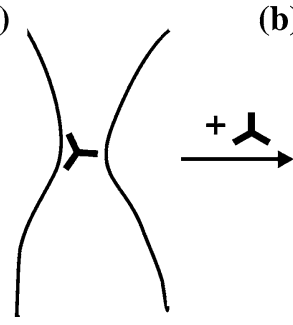

(b)

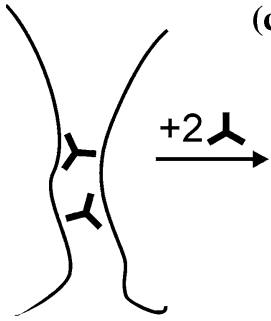

(c)

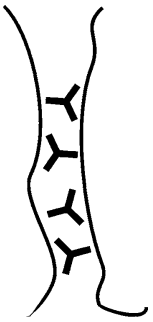

Fig. 9 Schematic representation of the "zipper-like" formation of domains of polyelectrolyte due to physical cross-linking by tristar-shaped TAM counterions (indicated by tristars, the detailed molecular structure is shown in Scheme 1). a The first TAM ion connects two polyelectrolyte chains or two domains of the same chain. b Close to the attachment site of the first ion, a favorable site for attachment of the second ion is formed. $\mathbf{c}$ Two chains or chain segments are now nearly parallel and predisposed to take up further TAM ions. Adapted from Ref. [23] with permission from Springer

organic TAM ions (with 12 hydroxyethyl side chains) with the hydrocarbon backbone of PDADMAC.

The picture sketched above somewhat resembles formation of thermoreversible gels, which has been studied extensively $[1,57,58]$. It is generally acknowledged to be a two-step process of initial creation of small macromolecular aggregates and subsequent further aggregation of these molecular clusters. Despite the heterogeneity of the two-step process, during gel-formation the system remains macroscopically homogeneous [1]. Although the situation is somewhat different in the case of TAM/ PDADMAC, one may nonetheless deduce that in analogy to the aggregated and nonaggregated states in thermoreversible gels, in the investigated system there may be isolated and clustered TAM crosslinks at any time, even at low ratios of spin probe/ polyelectrolyte. The overall system remains homogeneous on long length scales.

\section{Summary and Outlook}

We have shown how a broad variety of EPR spectroscopic methods can be used to investigate polyion-counterion interactions in polyelectrolyte solutions. The fact is exploited that EPR techniques are local methods, and by employing spin-carrying (i.e., EPR-active) probe ions, it is possible to examine polyelectrolytes from the counterions' point of view.

It was possible to gain insight into (a) the dynamics and local geometry of counterion attachment, (b) conformations and dynamics of local segments of the polyion in an indirect manner, and (c) the spatial distribution of spin probe ions that surround polyions in solution.

DEER measurements of FS ions were carried out to probe the distribution of attached counterions along polyelectrolyte chains. For a significant fraction of FS spin probes in solution with rigid rod model polyelectrolytes containing charged $\mathrm{Ru}^{2+}$ centers, a distance distribution was found that nicely reproduced the spacings of direct and next-neighbor $\mathrm{Ru}^{2+}$ centers along the polyelectrolytes. From the analysis of DEER data of FS/PDADMAC, it is suggested that PDADMAC chains 
form locally elongated structures of a size (i.e., persistence length) of at least $\sim 5 \mathrm{~nm}$.

Analysis of CW EPR spectra of dianion nitroxide spin probe Fremy's salt (FS, potassium nitrosodisulfonate) in solutions of cationic PDADMAC polyelectrolyte revealed that FS ions and PDADMAC form transient ion pairs with a lifetime of less than $1 \mathrm{~ns}$. This effect was called dynamic electrostatic attachment. By spectral simulation taking into account the rotational dynamics as an uniaxial Brownian reorientation, also the geometry of the attached state could be characterized. By variation of solvent, the effect of solvent viscosity and permittivity was investigated and indirect information of the polyelectrolyte chain motion was obtained.

Broadenings in CW EPR spectra of FS ions can be assigned to spin-exchange interaction and hence contain information on the local concentrations and distributions of the counterions. From the analysis of these broadenings in terms of a modified cylindrical cell approach (CCCM) of polyelectrolyte theory, radial distribution functions for the FS ions in the different solvents were obtained. This approach breaks down in water above a threshold value of $R(R=c$ (spin probe)/ $c$ (PDADMAC)), which again indicates that PDADMAC chain conformations are altered as a function of $R$.

When using large, tristar-shaped organic molecules of the triarylmethyl (TAM) class as trivalent tracer ions for PDADMAC chains, the network formation abilities of these large ions can be studied. By simulation of high-field/high-frequency ESEdetected EPR spectra, information on the distance distribution of TAM radicals within frozen solutions of PDADMAC could be obtained. The findings suggest that the large organic TAM spin probes can act as network-inducing agents in solutions of PDADMAC polyelectrolyte. Taking into account the topology and intrinsic stiffness of the polyelectrolyte chains, a "zipper-like" cooperative binding effect of TAM ions can explain such network formation.

On a more general level we have shown that the vast variety of EPR-based methods are powerful tools to understand complex, electrostatically self-assembled systems such as polyelectrolytes in solution and in networks. EPR spectroscopy can be seen as almost completely complementary to the widely used scattering methods in soft matter research since it solely characterizes the molecular level on a length scale up to $8 \mathrm{~nm}$ and time scales from the pico- to the microsecond regime.

This approach can be taken one step further using not only EPR-active counterions but also by spin-labeling the polyion chain. This will provide information that is complementary to that gained by the approach presented in this article.

Acknowledgments We thank Christian Bauer for technical support and Kurt Kremer, Christian Holm, and Manfred Schmidt for helpful discussions. We are also grateful to Nycomed Innovations AB, Sweden (now part of General Electric), for generously supplying us with the TAM radical. Financial support by the Deutsche Forschungsgemeinschaft (DFG) is gratefully acknowledged (priority program "High-Field EPR in Biology, Chemistry and Physics" (SPP 1051) and grant no. HI 1094/2-1).

Open Access This article is distributed under the terms of the Creative Commons Attribution Noncommercial License which permits any noncommercial use, distribution, and reproduction in any medium, provided the original author(s) and source are credited. 


\section{References}

1. H. Dautzenberg, W. Jaeger, J. Kötz, B. Philip, C. Seidel, D. Stscherbina, PolyelectrolytesFormation, Characterization and Application (Carl Hanser Verlag, Munich, 1994)

2. F. Oosawa, Polyelectrolytes (Marcel Dekker Inc., New York, 1971)

3. S. Förster, M. Schmidt, Adv. Polym. Sci. 120, 51 (1995)

4. G. Decher, Science 277, 1232 (1997)

5. S. Kumar, R. Nussinov, ChemBioChem 3, 604 (2002)

6. J. Kötz, S. Kosmella, T. Beitz, Prog. Polym. Sci. 26, 1199 (2001)

7. S. Ogawa, E.A. Decker, D.J. McClements, J. Agric. Food Chem. 51, 2806 (2003)

8. S.H. Lee, Polym. J. 32, 716 (2000)

9. M. Rubinstein, R.H. Colby, Polymer Physics (Oxford University Press, Oxford, 2003)

10. Y. Kantor, M. Kardar, Phys. Rev. E 51, 1299 (1995)

11. A.V. Dobrynin, M. Rubinstein, S.P. Obukhov, Macromolecules 29, 2974 (1996)

12. H.J. Limbach, C. Holm, K. Kremer, Europhys. Lett. 60, 566 (2002)

13. S. Minko, A. Kiriy, G. Gorodyska, M. Stamm, J. Am. Chem. Soc. 124, 3218 (2002)

14. T. Radeva (ed.), Physical Chemistry of Polyelectrolytes (Marcel Dekker Inc., New York, 2001)

15. N.M. Atherton, Principles of Electron Spin Resonance (Ellis Horwood, New York, 1993)

16. D. Hinderberger, G. Jeschke, H.W. Spiess, Macromolecules 35, 9698 (2002)

17. H.Q. Xue, P. Bhowmik, S. Schlick, Macromolecules 26, 3340 (1993)

18. G. Tsagaropoulos, J.S. Kim, A. Eisenberg, Macromolecules 29, 2222 (1996)

19. V. Schädler, A. Franck, U. Wiesner, H.W. Spiess, Macromolecules 30, 3832 (1997)

20. S.E. Cramer, G. Jeschke, H.W. Spiess, Macromol. Chem. Phys. 203, 182 (2002)

21. D. Hinderberger, H.W. Spiess, G. Jeschke, Macromol. Symp. 211, 71-86 (2004)

22. D. Hinderberger, H.W. Spiess, G. Jeschke, J. Phys. Chem. B 108, 3698-3704 (2004)

23. D. Hinderberger, G. Jeschke, H.W. Spiess, Colloid Polym. Sci. 282, 901-909 (2004)

24. D. Hinderberger, O. Schmelz, M. Rehahn, G. Jeschke, Angew. Chem. 116, 4716-4721 (2004); Angew. Chem. Int. Ed. 43, 4616-4621 (2004)

25. D. Hinderberger, H.W. Spiess, G. Jeschke, Europhys. Lett. 70, 102-108 (2005)

26. D. Hinderberger, G. Jeschke, Site-Specific Characterization of Structure and Dynamics of Complex Materials by EPR Spin Probes, Modern Magnetic Resonance: Materials Science (Springer, 2006)

27. V.M. Prabhu, Curr. Opin. Colloid Interface Sci. 10, 2-8 (2005)

28. V.M. Prabhu, B.D. Vogt, W.L. Wu, J.F. Douglas, E.K. Lin, S.K. Satija, D.L. Goldfarb, H. Ito, Langmuir 21, 6647-6651 (2005)

29. V.M. Prabhu, M. Muthukumar, G.D. Wignall, Y.B. Melnichenko, J. Chem. Phys. 119, 4085-4098 (2003)

30. A. Schweiger, G. Jeschke, Principles of Pulse Electron Paramagnetic Resonance (Oxford University Press, Oxford, 2001)

31. G. Manning, J. Chem. Phys. 51, 924 (1969)

32. G. Manning, J. Chem. Phys. 51, 934 (1969)

33. G. Manning, Acc. Chem. Res. 12, 443 (1979)

34. G. Manning, J. Chem. Phys. 89, 3772 (1988)

35. M. Deserno, Ph.D. Thesis, Max-Planck Institute for Polymer Research, Mainz, Germany (2000)

36. A. Deshkovski, S. Obukhov, M. Rubinstein, Phys. Rev. Lett. 86, 2341 (2001)

37. S. Kelch, M. Rehahn, Macromolecules 32, 5818 (1999)

38. O. Schmelz, M. Rehahn, e-Polymers 47 (2002)

39. C. Wandrey, J. Hernandez-Barajas, D. Hunkeler, Adv. Polym. Sci. 145, 123 (1999)

40. S.T. Dubas, J.B. Schlenoff, Macromolecules 32, 8153 (1999)

41. G. Jeschke, A. Koch, U. Jonas, A. Godt, J. Magn. Reson. 155, 72-82 (2002)

42. A.D. Milov, A.G. Maryasov, Y.D. Tsvetkov, Appl. Magn. Reson. 15, 107-143 (1998)

43. A.D. Milov, A.B. Ponomarev, Y.D. Tsvetkov, Chem. Phys. Lett. 110, 67 (1984)

44. B.E. Bode, D. Margraf, J. Plackmeyer, G. Durner, T.F. Prisner, O. Schiemann, J. Am. Chem. Soc. 129, 6736-6745 (2007)

45. Y.N. Molin, K.M. Salikhov, K.I. Zamaraev, Spin Exchange-Principles and Applications in Chemistry and Biology. Springer Series in Chemical Physics, vol. 8 (Springer-Verlag, Berlin, 1980)

46. M.T. Jones, J. Chem. Phys. 38, 2892 (1963)

47. M.P. Eastman, G.V. Bruno, J.H. Freed, J. Chem. Phys. 52, 2511 (1970) 
48. D.J. Schneider, J.H. Freed, in Biological Magnetic Resonance, vol. 8, chap. 1, ed. by L.J. Berliner, J. Reuben (Plenum Press, New York, 1989)

49. M. Pannier, S. Veit, A. Godt, G. Jeschke, H.W. Spiess, J. Magn. Reson. 142, 331 (2000)

50. J.S. Hyde, M. Pasenkiewicz-Gierula, A. Jesmanowicz, W.E. Antholine, Appl. Magn. Reson. 1, 483 (1990)

51. G. te Velde, F.M. Bickelhaupt, E.J. Baerends, C. Fonseca Guerra, S.J.A. van Gisbergen, J.G. Snijders, T. Ziegler, J. Comput. Chem. 22, 93 (2001)

52. T. Ichikawa, L. Kevan, M.K. Bowman, S.A. Dikanov, Y.D. Tsvetkov, J. Chem. Phys. 71, 1167 (1979)

53. J.H. Ardenkjaer-Larsen, I. Laursen, I. Leunbach, G. Ehnholm, L.G. Wistrand, J.S. Petersson, K. Golman, J. Magn. Reson. 133, 1 (1998)

54. L. Yong, J. Harbridge, R.W. Quine, G.A. Rinard, S.S. Eaton, G.R. Eaton, C. Mailer, E. Barth, H.J. Halpern, J. Magn. Reson. 152, 156 (2001)

55. W. Dawydoff, K.J. Linow, B. Philipp, Acta Polym. 42, 592, 646 (1991)

56. C.T. Farrar, D.A. Hall, G.J. Gerfen, M. Rosay, J.H. Ardenkjaer-Larsen, R.G. Griffin, J. Magn. Reson. 144, 134 (2000)

57. D.A. Rees, Carbohydr. Chem. 24, 267 (1969)

58. W. Dawydoff, K.J. Linow, B. Philipp, Nahrung 28, 241 (1984)

59. G. Jeschke, M. Sajid, M. Schulte, A. Godt, Phys. Chem. Chem. Phys. 11(31), 6580-6591 (2009) 